Article

\title{
Computational Modeling Methods for Deployable Structure Based on Alternatively Asymmetric Triangulation
}

\author{
Qingwen Zhang ${ }^{1,2, *}$, Danmin $\mathrm{Yu}^{1,2}$, Xinye $\mathrm{Li}^{1,2}$ and Feng Fan ${ }^{1,2}$ \\ 1 Key Lab of Structures Dynamic Behavior and Control of the Ministry of Education, Harbin Institute of \\ Technology, Harbin 150090, China; 18s033064@stu.hit.edu.cn (D.Y.); xinyeli@tongji.edu.cn (X.L.); \\ fanf@hit.edu.cn (F.F.) \\ 2 Key Lab of Smart Prevention and Mitigation of Civil Engineering Disasters of the Ministry of Industry and \\ Information Technology, Harbin Institute of Technology, Harbin 150090, China \\ * Correspondence: zhangqw@hit.edu.cn
}

Received: 22 September 2019; Accepted: 10 October 2019; Published: 11 October 2019

check for updates

\begin{abstract}
Asymmetric triangulation is an interesting method combined with concentric pleating to obtain a 3D shape without stretching or tearing. There exists some geometric properties in the process of folding to help realize extension and contraction, which can be used in parametric modeling of different regular polygons. To facilitate design and modeling, adequate computational modeling methods are indispensable. This paper proposes a new mathematical idea and presents a feasible way to build the parameterized models in the digital environment of Rhinoceros, utilizing the Kangaroo plugin in Grasshopper. Designers can directly observe the models' kinematic deployment and calculate the folding efficiency. It is concluded that the tendencies of folding efficiency in different regular polygons are not the same. To realize rigid folding, each polygon has a limited folding angle.
\end{abstract}

Keywords: deployable structure; regular polygon; alternatively asymmetric triangulation; parametric modeling; rigid-foldable origami

\section{Introduction}

Deployable structures, also known as pliable structures, were initially found in the design of umbrellas. It is known that deployable structures are a kind of structure with a stable bearing capacity made up of prefabricated elements which are able to be deployed from the initial small-volume configuration to the unfolded condition according to the predesign. In most cases, these elements move along the fixed track to generate a folding motion [1]. To change the configuration from flat sheets to curved geometries, this process can find reference experience from a well-known Japanese art form, origami, which makes use of flat paper to obtain a three-dimensional shape without stretching or tearing. These macroscale bending and folding techniques can be used to fabricate micro- and nano-structured devices [2,3] such as electronic circuits, sensors, antennas, and biomimetic constructs (Figure 1). On the macro scale, the idea of folding and bending inspires people to design novel kinetic systems for architectural applications [4], especially for those applied in space exploration, including various booms [5] and expandable bases on Moon or Mars [6]. Besides, this novel idea is conducive to the development of new materials [6] that are practicable in folding and bending. 


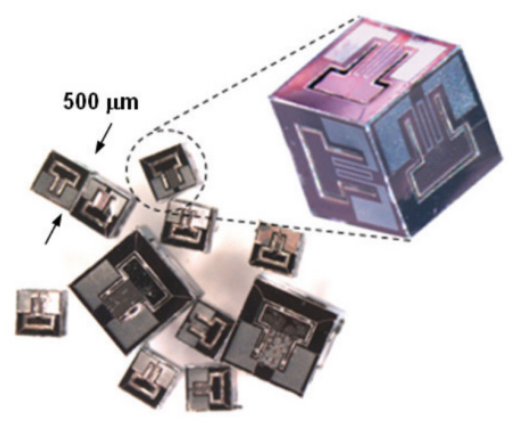

(a)

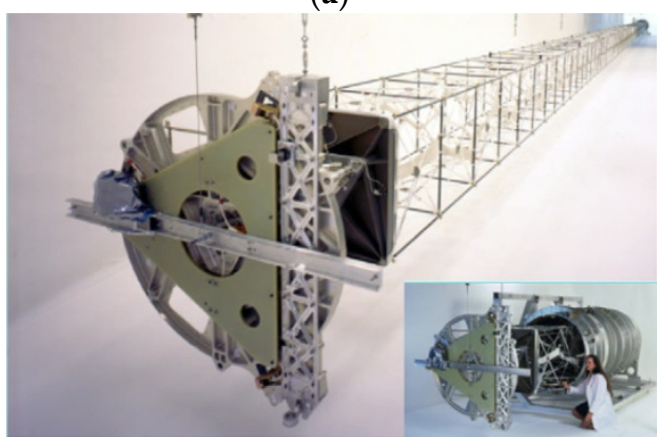

(c)
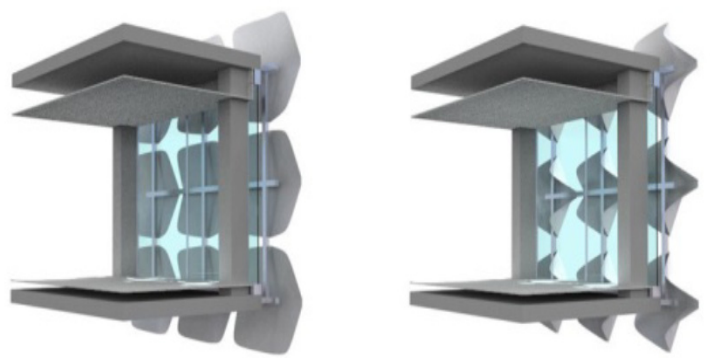

(b)

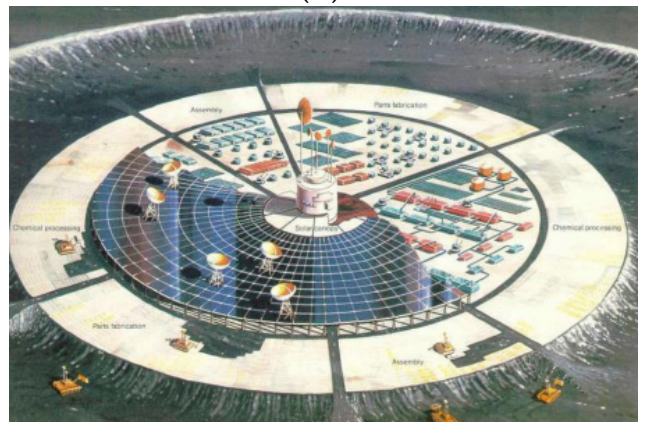

(d)

Figure 1. Examples of deployable structures tht are used: (a) Optical images showing experimentally realized packaging of cantilever sensors and magnetic field sensitive strain gauges in polyhedral geometries [3]; (b) conceptual design of a facade with adaptive shading elements based on curved-line folding [7]; (c) the ADAM mast deployed and canister [8]; (d) expandable base (courtesy of NASA) [6].

With a long history, origami functions not just as an art form, but as an inspiration for engineering and mathematics as well. Origami using non-singular patterns includes various types of folding methods that have one single mid-point and can be divided into sub-patterns rotating around the mid-point [9]. Tachi et al. summarized the rule of these patterns' degree of freedom [10,11]. Miura predicted one configuration which was later named the developable double corrugation surface (DDC surface), also called Miura-ori [12]. Its symmetry has been discussed by Sareh et al. [13] and different kinds of derivation from Miura-ori were presented by Sareh and Guest [14]. Their research has confirmed that this rigid fold pattern theoretically has a single degree of freedom $[15,16]$. Unlike Miura-ori, whose cells are an in-line arrangement to make the contraction and expansion in rectilinear directions, Resch-ori tessellation consists of regular-polygon cells arranged circularly [17]. This idea can be applied to the configuration of free-form surfaces, but there are more difficulties in building this tessellation and keeping it stable. Besides, Tachi proposed an origami approach with the idea of tucking molecules to construct a desired polyhedral surface by folding a single sheet of material [18].

Concentric pleating, which also has a lot to do with symmetry, can be traced back to the Bauhaus School in the 1920s and its popularity was attributed to an origami artist named ThokiYenn. Structures following this pattern use sunk creases (valleys, which are presented as dotted lines in figures) and bulgy creases (mountains, which are presented as full lines in figures) alternately to build deployable negative Gauss surfaces. This pattern can be applied in regular polygons, as shown in Figure 2, and even circular planes. 


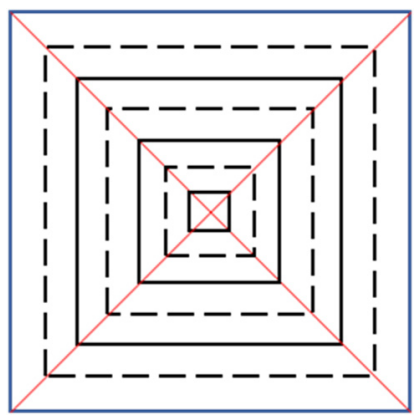

(a)

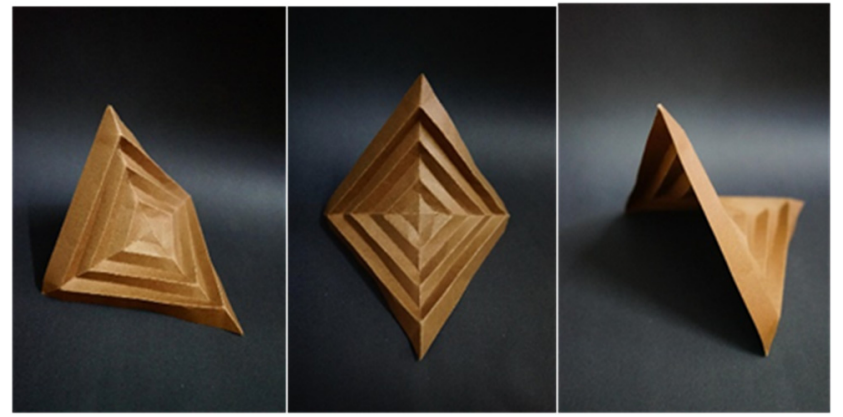

(b)

Figure 2. Square concentric pleating origami and the crease pattern: (a) The crease pattern; (b) the origami model.

The negative Gauss surface obtained by concentric pleating is produced by torsion of a series of closed strip loops and these closed-loop creases generate a curved surface, while linear creases help build planar folding. Demaine et al. [19] proved that it is impossible to achieve folds without stretching, tearing, or introducing additional creases because of the torsion inside each small trapezoid [19]. They were inspired by Miura-ori to add one more crease inside each tetragon to obtain rigid folding, as shown in Figure 3 (the black lines represent the original creases of which the full lines are mountains and the dotted lines are valleys, and the creases added into each tetragon are shown in red lines), and to create the two types of foldable triangulation.

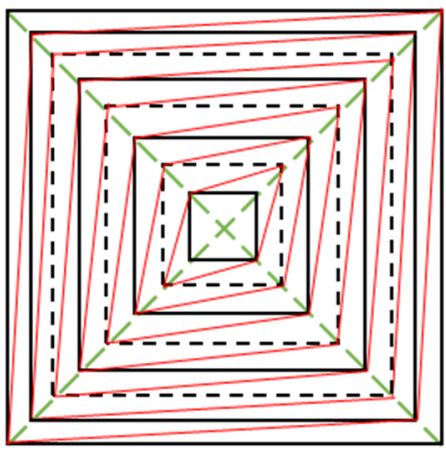

(a)

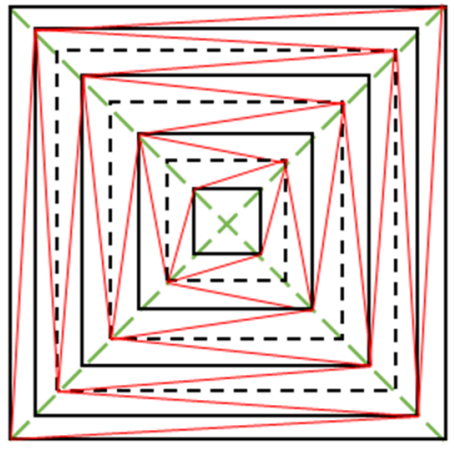

(b)

Figure 3. Foldable triangulations of the hyperbolic paraboloid crease pattern: (a) Asymmetric triangulation; (b) alternating asymmetric triangulation.

Based on the properties of squares using concentric pleating, this paper chooses concentric pleating (alternating asymmetric triangulation), applies this pattern to the parametric modeling of regular hexagons and generalizes it to n-regular-polygons, because of its high folding efficiency. Following this, we analyze the efficiency, static performance and the rigid folding rule in this folding pattern. Finally, conclusions are drawn regarding the pattern of tessellation consisting of deployable regular polygons.

\section{Concentric Pleating in Tetragons And Hexagons}

\subsection{Two Categories of Concentric Pleating}

The concepts of "mountains" and "valley" also work on the standard crease pattern for concentric pleating, referring to different creases, as shown in Figure 2, with two line-types. The standard folding pattern fails to obtain rigid folding and brings about additional creases, which are marked in Figure 4, and stretching or tearing. It is obvious that when the fold angle $\theta$ reaches the limit, there will be torsion and buckling inside these trapezoids and the boundary lines show the maximum deformation. Inside 
every single trapezoid, the maximum deformation exists along its diagonal and this helps make a conjecture that if the trapezoid is divided by its diagonal into two triangles, it is probable that a rigid fold pattern exists.

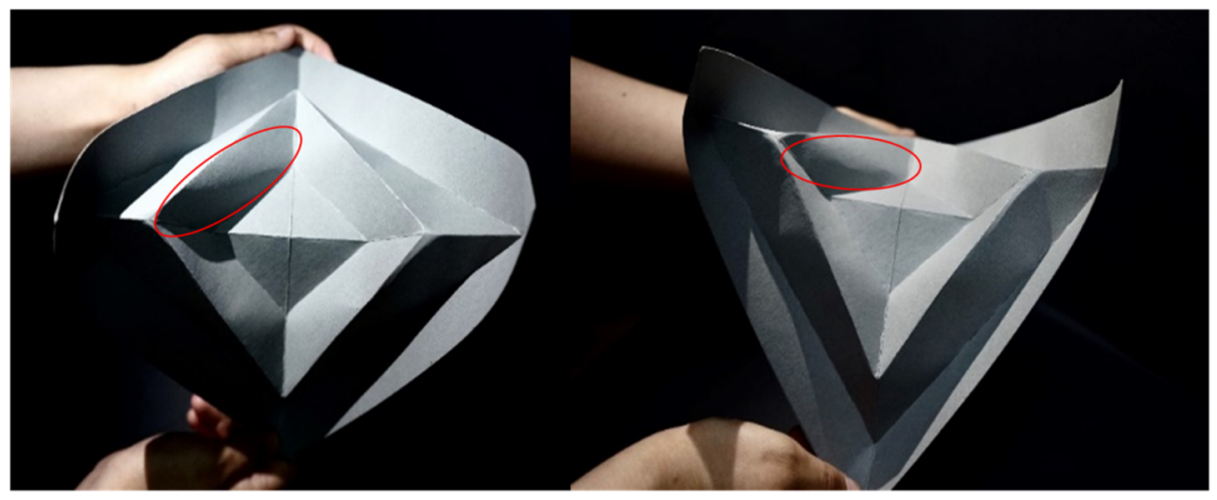

Figure 4. Test for concentric pleating of squares.

These properties also work in the concentric pleating of hexagons which leads to special negative Gauss surfaces and a non-rigid folding structure, as shown in Figure 5.

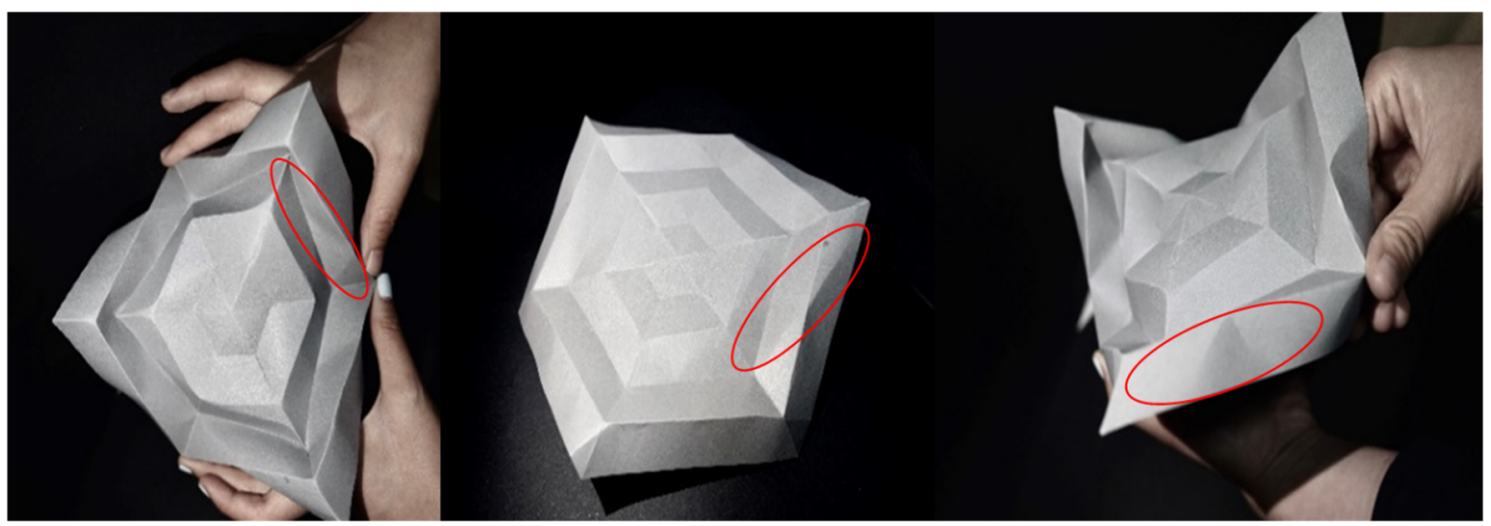

Figure 5. Test for concentric pleating of regular hexagons.

It has been mentioned that triangulation is a possible approach to help realize rigid fold and Demaine et al. used it to propose two resolutions [19] to this problem, called asymmetric triangulation and alternating asymmetric triangulation (Figure 3). Within a certain range of the fold angle, there is no torsion or buckling, which means that triangulation is an effective way to obtain rigid folds of squares and this result also works on regular hexagons. Whether they are squares or regular hexagons, alternating asymmetric triangulation has higher folding efficiency than asymmetric triangulation, so in the following parts this paper focuses on the former method for further discussion.

The degrees of freedom (DOF) of alternating asymmetric triangulation conforms to the rule of Formula (1); thus, for regular tetragons, the degree of freedom is one and for regular hexagons, it is three.

\subsection{Parametric Modeling}

\subsubsection{Geometrical Relationship in Squares}

Here, the geometrical properties of squares using alternating asymmetric triangulation are reviewed.

It is assumed that one diagonal of the central square is fixed on the $y$-axis and points $a_{i}$ and $b_{i}$ $(i=1,2, \ldots)$ will move on the $Y-Z$ plane and $X-Z$ plane, respectively. Here, the way this paper finds 
these vertexes will be illustrated with examples of $a_{2}$ and $b_{2}$, as shown in Figure 6 . The width of one loop is defined as $d$, so the length of these creases are

$$
\begin{gathered}
a_{0} a_{1}=a_{0} b_{1}=d / \cos 45^{\circ}, \\
a_{1} a_{2}=b_{1} b_{2}=d / \cos 45^{\circ}, \\
a_{1} b_{1}=2 * d, \\
a_{2} b_{1}=\sqrt{a_{0} b_{1}^{2}+\left(a_{0} a_{1}+a_{1} a_{2}\right)^{2}} .
\end{gathered}
$$

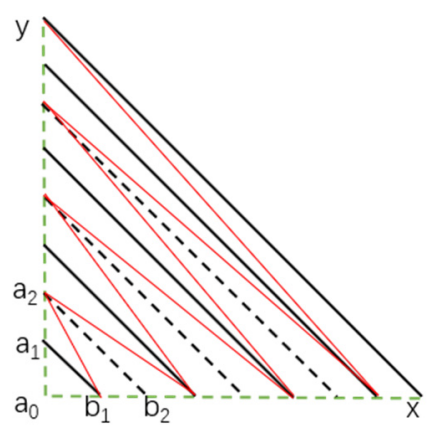

Figure 6. A sub-plate of a square with alternative asymmetric triangulation.

A sphere is modeled whose center is a1 and the radius is the length of $a_{1} a_{2}$, while the intersection of the sphere and $\mathrm{Y}-\mathrm{Z}$ plane is a circle. Similarly, there is a sphere of radius $\mathrm{a}_{2} \mathrm{~b}_{1}$ centered at $\mathrm{b}_{1}$ intersecting with the $\mathrm{Y}-\mathrm{Z}$ plane so that another circle is obtained on this plane, as shown in Figure 7.

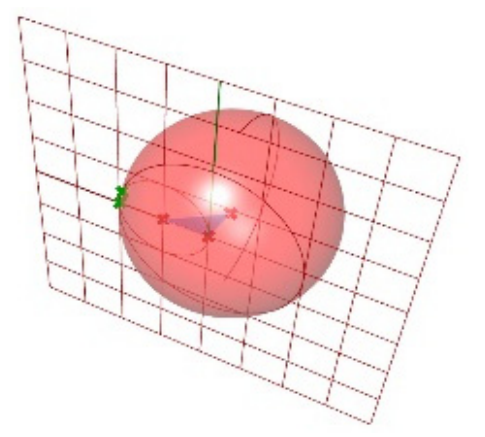

Figure 7. Intersection of a sphere and a given plane.

The intersection of two circles will be two different points, one single point or nothing. Here we assume that there must be one or two points in the intersection of these two circles on the $\mathrm{Y}-\mathrm{Z}$ plane. If the solution is one point, then this solution is the genuine one, the coordinates of $a_{1}$. When there are two different solutions, as shown in Figure 8, the real solution can be easily confirmed because this surface has a saddle-shaped configuration and if $\mathrm{a}_{0} \mathrm{a}_{1}$ is fixed, then $\mathrm{a}_{1}$ must be below the $\mathrm{X}-\mathrm{Y}$ plane, which means that its y coordinate is a positive number. By this approach, the coordinates of $b_{2}$ are at the intersection of two circles on the $X-Z$ plane. The two circles are intersections of two different spheres of radii $a_{2} b_{2}$ and $b_{1} b_{2}$ centered at $a_{2}$ and $b_{2}$, respectively, and this saddle-shaped surface makes this point $b_{2}$ located above the $X-Y$ plane. Demaine et al. used the mountain-valley assignment to determine the genuine solution and propose an algorithm based on Mathematica's fully expanded solution for the general case to obtain this structure [10]. 


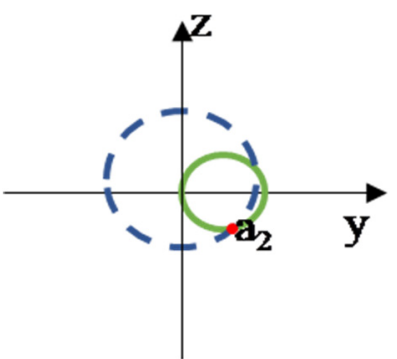

(a)

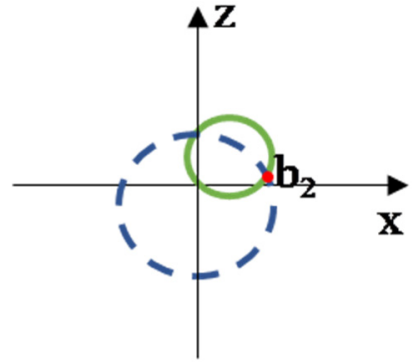

(b)

Figure 8. Using the intersections of circles on the different co-ordinate planes to find $a_{2}$ and $b_{2}$ : (a) Intersections of circles on the $\mathrm{Y}-\mathrm{Z}$ plane; (b) intersections of circles on the $\mathrm{X}-\mathrm{Z}$ plane.

The coordinates of other points can be calculated step by step and the location of $b_{1}$ is only decided by the folding angle, which is the basic parameter. When the sub-plate is completed, the whole plate can be constructed by copying the sub-plate itself, as shown in Figure 9.
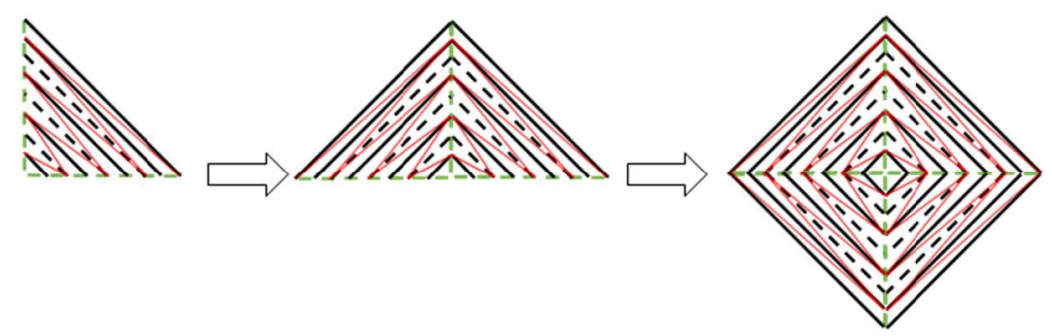

Figure 9. How to complete the whole plane based on the calculation of the sub-plane.

\subsubsection{Geometrical Relationship in Regular Hexagons}

Since former researches mainly focus on the alternating asymmetric triangulation of squares and have given narration on the modeling [19], this paper generalizes this triangulation to regular hexagons and even n-regular polygons. However, this differs from the modeling of squares. In the demonstration before, it is clear that $a_{0} a_{1}$ can be assumed as fixed, while for regular hexagons only the mid-point can be regarded as an immobile one, which leads to a new geometric relationship.

In the initial state, the whole plate is flat and the following part illustrates some eternal geometric rules to help the construction of the parametric model. The design process mainly focuses on the geometrical relationship in a sub-plate and the final crease pattern can be created by symmetrically coping itself.

During kinematic motion, the distances between each pair of adjacent points are fixed, which this paper makes use of to build the model. In a regular hexagon, the central point, $a_{0}$, is permanent, and to obtain the coordinates of the other points there are some rules that can be followed [19].

In Figure 10, it is assumed that the mid-point $a_{0}$ is fixed at the original and $a_{i}(i=1,2, \ldots)$ is moving within the $\mathrm{Y}-\mathrm{Z}$ plane. The point $\mathrm{b}_{1}$ is regarded as rotating around the m-axis, the angle between this and the y-axis being $30^{\circ}$. Thus, the folding angle refers to the angle that $a_{0} b_{1}$ is rotating around the $\mathrm{m}$-axis and, once the angle is given, the length of $a_{0} b_{1}$ can be derived from $\mathrm{d}$ and the regular triangle's geometric properties.

$$
\begin{gathered}
\angle a_{1} a_{0} b_{1}=60^{\circ}, \\
a_{0} a_{1}=a_{0} b_{1}=a_{1} a_{2}=a_{1} b_{1}=b_{1} b_{2}=d / \cos 60^{\circ}, \\
a_{2} b_{1}=2 * d .
\end{gathered}
$$




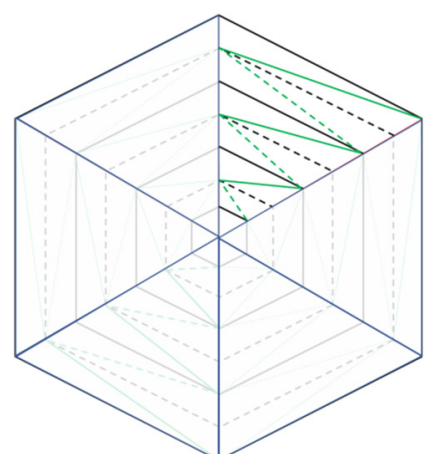

(a)

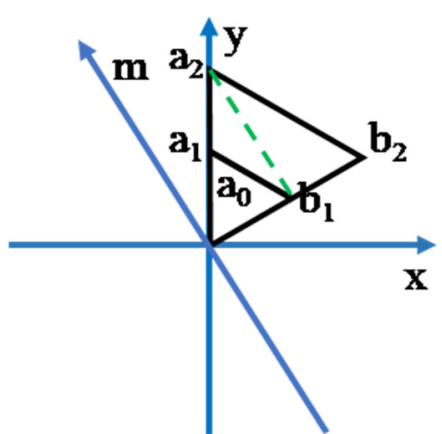

(b)

Figure 10. A regular hexagon using alternating asymmetric triangulation. (a) The creases of a regular hexagon, in which the full lines represent the mountains and the dotted lines represent the valleys in this folding pattern. The green lines are used to triangulate the trapezoids; (b) a sub-plate of a regular hexagon in the $X-Y$ plane.

These distances are immobile, so the coordinates of $a_{i}$ and $b_{i}$ are derived from them. This paper assumes that the initial state is when the paper is a flat sheet without deformation, as shown in Figure 11. Once the location of $b_{1}$ is obtained, a sphere with the radium of $a_{1} b_{1}$, whose center is $b_{1}$, will intersect with the $\mathrm{Y}-\mathrm{Z}$ plane to generate a circle on this plane. Then, $a_{1}$ is the solution of the intersection of this circle and another one which is centered at $a_{0}$ with a radius of $a_{0} a_{1}$ on this plane. When there are two solutions in the intersection, the genuine solution must follow the rules of a negative Gauss surface, as mentioned in Section 2.2.1. This process is shown in Figure 12.

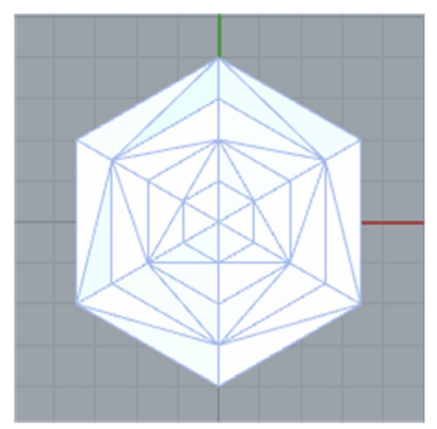

Figure 11. The initial state of a regular hexagon in Rhinoceros.
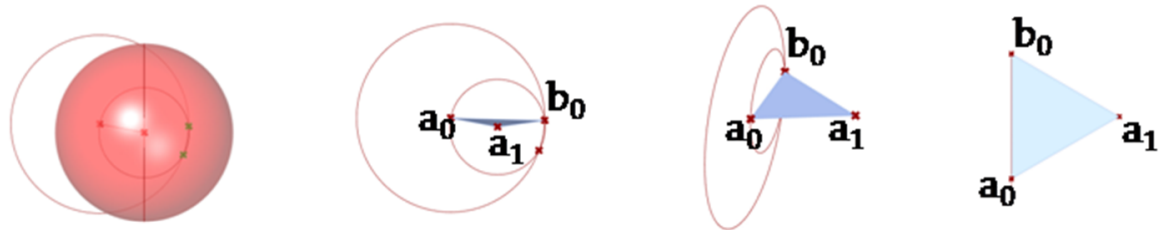

Figure 12. Diagrammatic drawing of how to locate $b_{0}$ and $a_{1}$.

After confirming the positions of the first triangulation of a hexagon, the following points' coordinates can be calculated with similar methods by intersecting several spheres, as shown in Figure 13. 

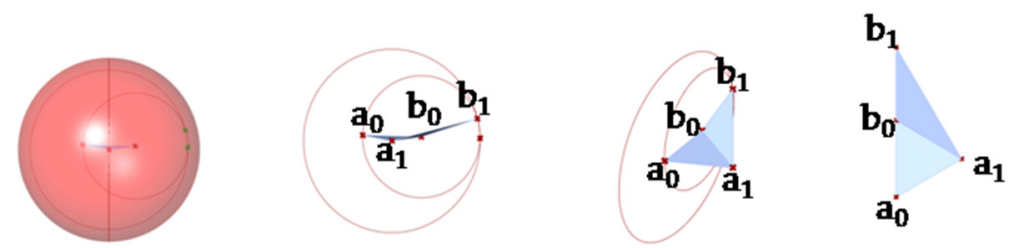

Figure 13. Diagrammatic drawing of how to locate $b_{1}$ with the change of the folding angle.

Figure 14 illustrates the process after one sub-plate of a hexagon is completed, where the symmetry of a regular hexagon with alternating asymmetric triangulation will help finish the model.
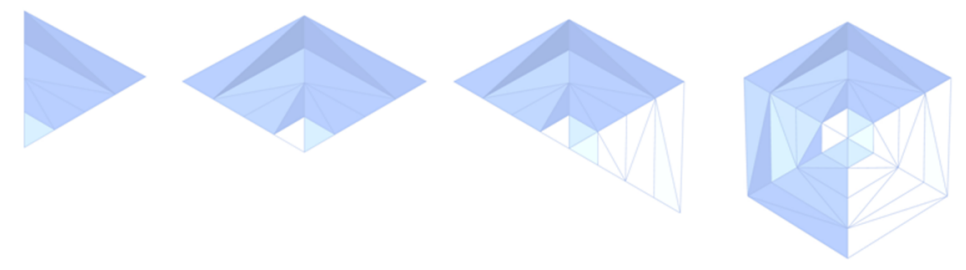

Figure 14. How to construct the model of a regular hexagon from a sub-plate.

These ideas about kinematic motion can be generalized to n-regular polygons for parametric modeling.

\subsubsection{Parametric Modeling of Deployable Structures Based on Alternating Asymmetric Triangulation}

Parametric modeling is a process that transfers the design itself into functions, and by adjusting the initial conditions these functions will automatically compute the results. Tachi et al. developed three programs named Rigid Origami Simulator [20], Orimamizer [21,22] and Freeform Origami [23] to help design origami approaches. This paper utilizes the Kangaroo [24] plugin in Grasshopper [25], developed by Daniel Piker, to build the parameterized models of these structures in the digital environment of Rhinoceros [26] according to the ideas above. Grasshopper is a graphical algorithm editor tightly integrated with Rhinoceros' 3-D modeling tools for designers who are exploring new shapes using generative algorithms. Meanwhile, Grasshopper and Rhinoceros combined together can realize visual modeling, which is within the trend of intellectualized design. Kangaroo is a Live Physics engine for interactive simulation, optimization, and form-finding directly within Grasshopper.

For the models discussed in this paper, the basic parameters are the fold angle of the central square, the width of each closed-loop, the number of edges of the polygons. According to Tachi [9], when the number of edges and the width of loops are fixed, the coordinates of vertices are available, so in the process of parametric modeling, this paper chooses the fold angle of the central square as the basic parameter. As shown in Figure 15, by adjusting one parameter related to the folding angle, the bar marked in Figure 15, the whole condition of this model can be changed. The specific model built in Grasshopper is presented in Appendix A. 


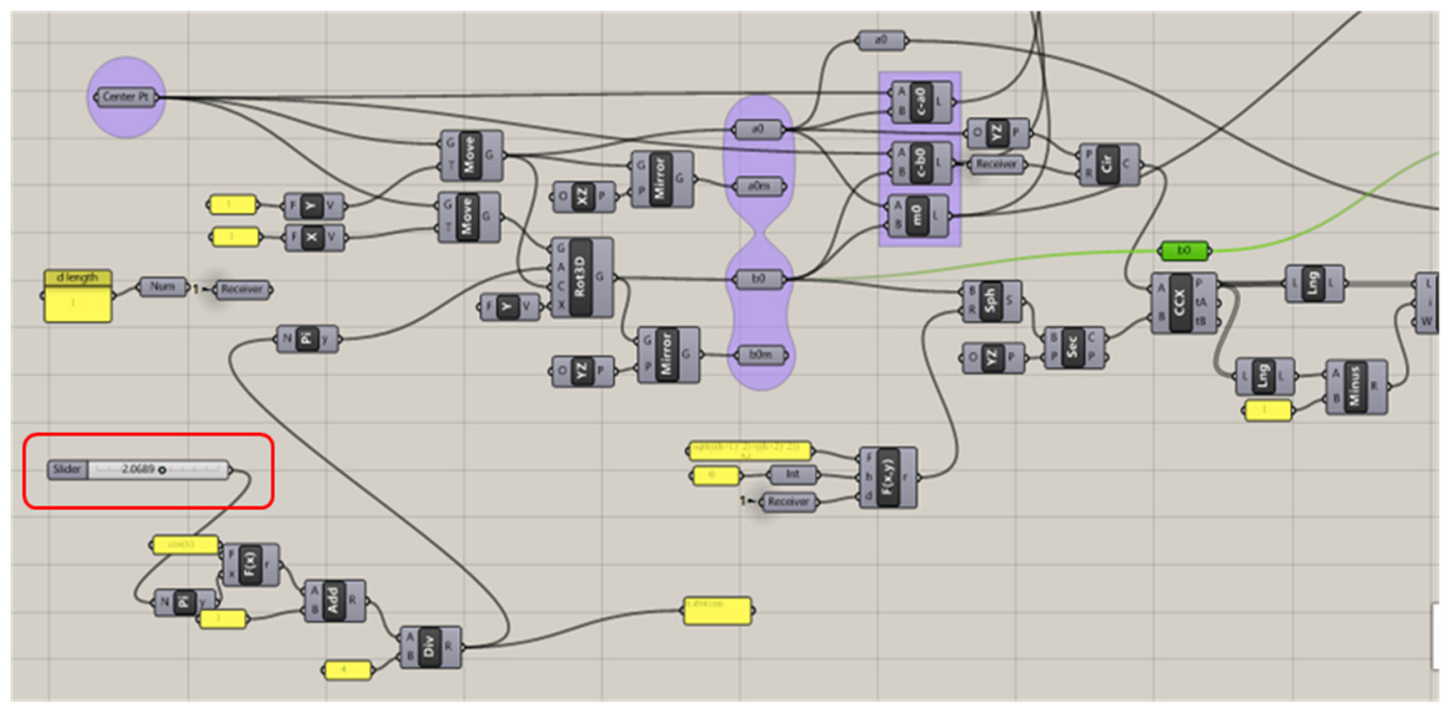

Figure 15. Demonstration of the use of Kangaroo in Grasshopper to model the folding motion of a pattern using alternating asymmetric triangulation.

The parametric model of squares and regular hexagons using alternating asymmetric triangulation is shown in Figure 16.

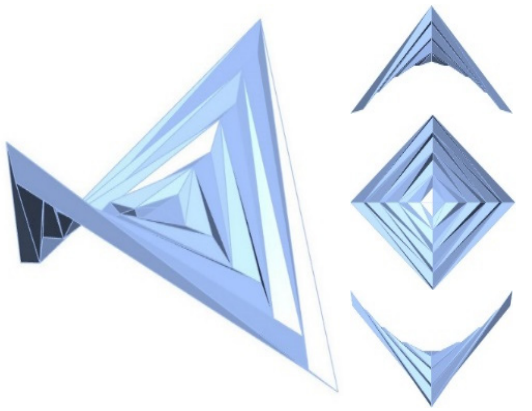

(a)

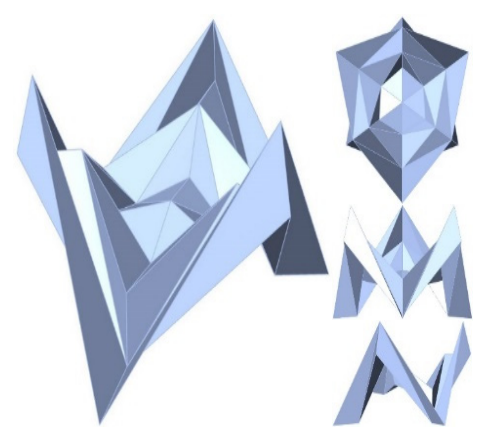

(b)

Figure 16. The parametric model of squares and regular hexagons with alternating asymmetric triangulation obtained in the digital environment of Rhinoceros: (a) 3-D models of squares; (b) 3-D models of regular hexagons.

In addition to modeling of squares and regular hexagons, the parametric modeling of n-regular polygons using alternating asymmetric triangulation is possible. Though there are some subtle differences among polygons with various number of edges, the geometric relationships among vertices and creases can be derived through the geometric properties. Here, this paper summarizes and gives the flow diagram of parametric modeling for the n-regular polygon, as shown in Figure 17. 


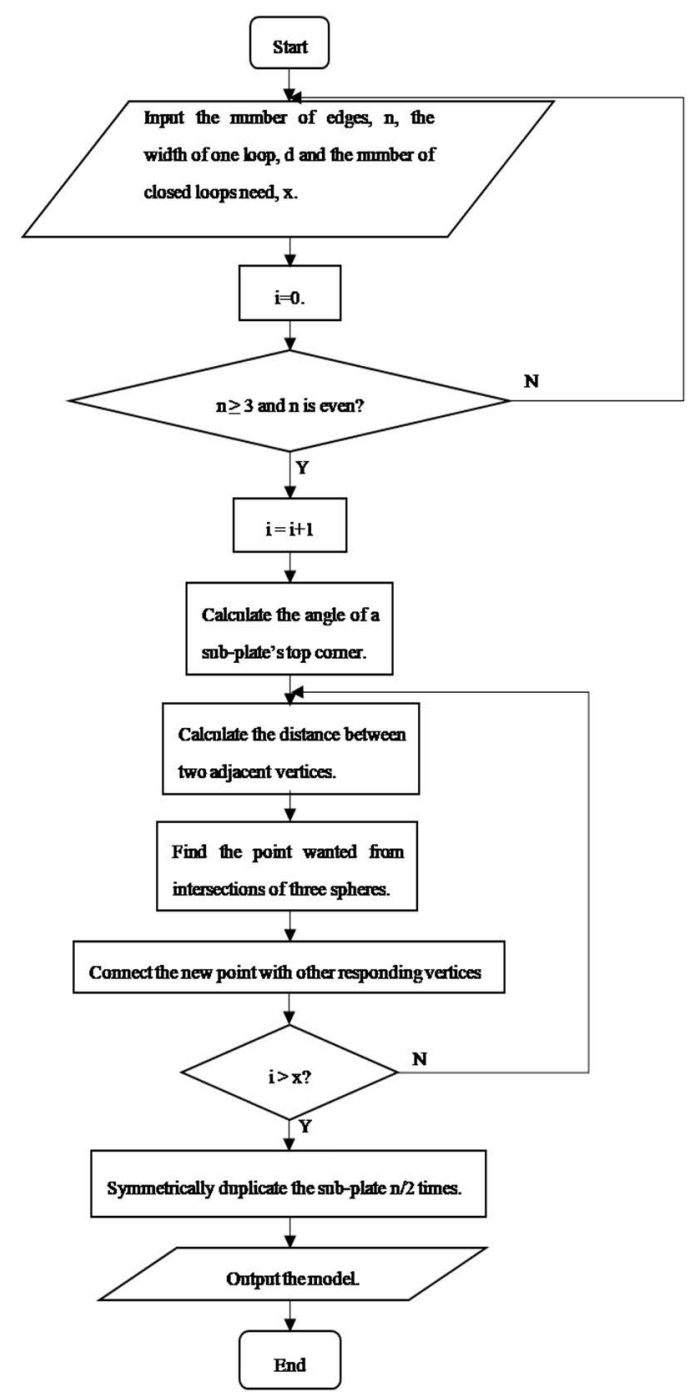

Figure 17. Flow chart of an n-regular polygon's alternating asymmetric triangulation.

\subsection{Analysis of Kinematics Motions}

To obtain the kinematic properties and the folding efficiency of alternating asymmetric triangulation, it is necessary to analyze deployable structures' kinematic motions. Using the models constructed in the platform of Rhinoceros, this paper will discuss the variation trend of the projected area of the top view and the height of the side view. This paper defines the maximum projected area and the maximum height during the process of deployment as the reference values to normalize these variables. The results are shown in Figure 18 , and $\mu_{1}$ and $\mu_{2}$ are defined as following to make it clearer:

$$
\begin{aligned}
& \mu_{1}=A_{1} / A_{2}, \\
& \mu_{2}=H_{1} / H_{2} .
\end{aligned}
$$

where $A_{1}$ refers to the projected area of the top view, $A_{2}$ is the maximum projected area, $H_{1}$ is the projected height of the side view, and $\mathrm{H}_{2}$ is the maximum projected height. 


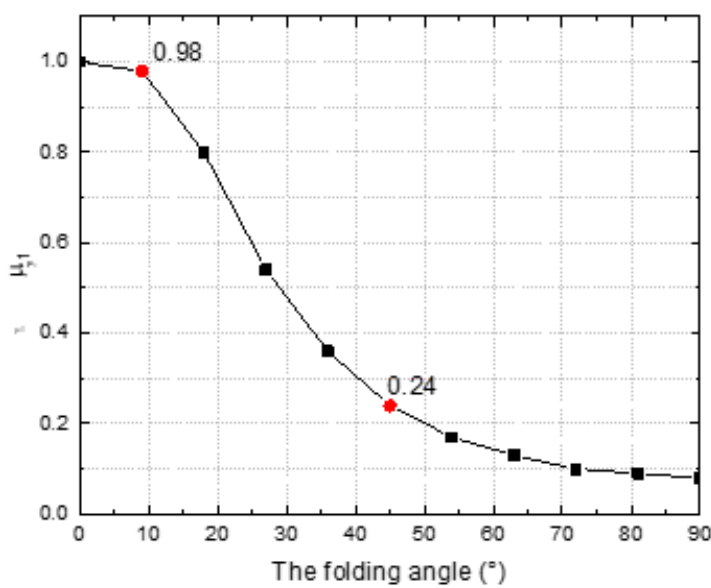

(a)

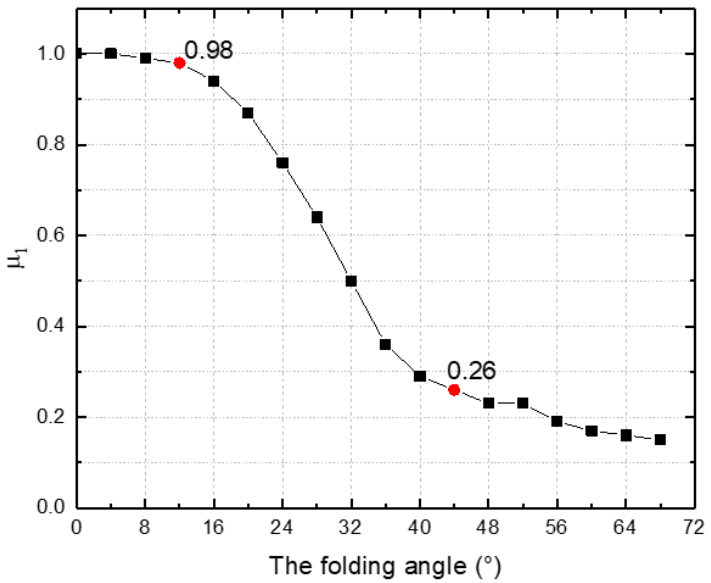

(c)

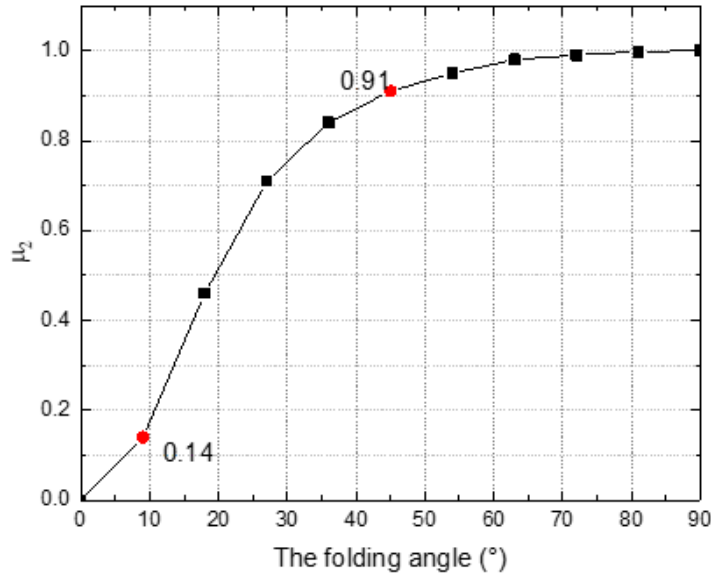

(b)

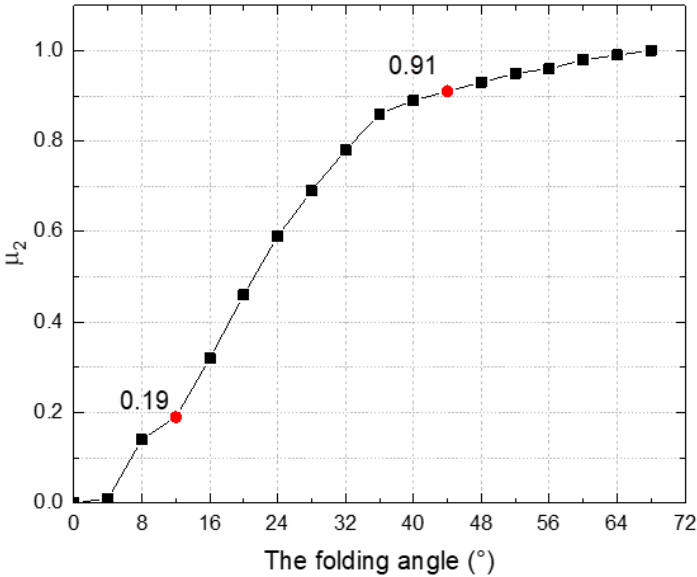

(d)

Figure 18. The trends of $\mu_{1}$ and $\mu_{2}$ in squares and regular hexagons: (a) The trend of $\mu_{1}$ in squares; (b) the trend of $\mu_{2}$ in squares; (c) the trend of $\mu_{1}$ in regular hexagons; (d) the trend of $\mu_{2}$ in regular hexagons.

It can be concluded that the relationships are obviously nonlinear for the surfaces as Gauss surfaces using these results for reference and observing the data from parametric analysis. The graphs of a regular tetragon show the characteristics of quadratic lines and the graphs of a regular hexagon demonstrate similarities with cubic curves.

Specifically, during the deployment of squares, the projected area of the top view experiences a sharp decline when the fold angle, $\theta$, is around $10^{\circ}$, and then a less steep decline around $45^{\circ}$; the same applies to hexagons. Consequently, it is possible that this trend is determined by this pattern's geometric properties and has nothing to do with the number of edges of the plate. Furthermore, it is likely that an n-regular polygon applying alternating asymmetric triangulation will illustrate these trends as well and the number of edges just affects the maximum fold angle. The more edges this plate has, the lower the fold angle it can reach.

\subsection{Rigid-Foldable Origami}

The research of Fushimi et al. [27] about the rigid-foldable origami of zero-thickness paper leads to the famous Fushimi Theorem, which shows the condition of angle that the folding pattern has to meet. From the crease patterns shown in Figures $3 b$ and $10 a$, the numbers of creases meeting in one vertex varies at different points, which is alternately four or six in the first closed loop in a square or a hexagon, and compared with the similar topological relationship, the variation follows some rules. It is obvious that the number of regular polygons' edges has to be even if the alternating asymmetric 
triangulation is feasible. Table 1 gives the distribution regularities of the number of creases in one single vertex in different regular polygons whose number of edges is even.

Table 1. Number of creases meeting in different vertices.

\begin{tabular}{ccccccc}
\hline Type of Polygons & $\begin{array}{c}\text { The Central } \\
\text { Point }\end{array}$ & $\begin{array}{c}\text { Points on } \\
\text { 1st Closed } \\
\text { Loop }\end{array}$ & $\begin{array}{c}\text { Points on } \\
\text { 2nd Closed } \\
\text { Loop }\end{array}$ & $\begin{array}{c}\text { Points on } \\
\text { 3rd Closed } \\
\text { Loop }\end{array}$ & $\ldots$ & $\begin{array}{c}\text { Points on } \\
\text { Edges }\end{array}$ \\
\hline Regular tetragon & 4 & 4 or 6 & 4 or 8 & 4 or 8 & 4 or 8 & 3 or 5 \\
Regular hexagon & 6 & 4 or 6 & 4 or 8 & 4 or 8 & 4 or 8 & 3 or 5 \\
Regular octagon & 8 & 4 or 6 & 4 or 8 & 4 or 8 & 4 or 8 & 3 or 5 \\
N-regular polygon & $\mathrm{n}$ & 4 or 6 & 4 or 8 & 4 or 8 & 4 or 8 & 3 or 5 \\
\hline
\end{tabular}

Through further study, it is found that when the point has a four-crease pattern, among the four creases, there will be three mountains and one valley, or three valleys and one mountain; when it is the six-crease pattern, there will be four mountains and two valleys, four valleys and two mountains. If there are eight creases meeting at one point, these creases always consist of five mountains and three valleys, or five valleys and three mountains. The regularity in the four-crease pattern is the aforementioned Fushimi Theorem.

\section{Discussion}

\subsection{The Maximum Foldable Angle of One Single Regular Polygon Using Alternating Asymmetric Triangulation}

One advantage which the visual modeling brings out is that users can observe the model which is built directly and make proper adjustments. As shown in Figure 19, the statements before and after the folding angle reaches its maximum value have significant differences. From Figure 19b, it is obvious that the statement of this hexagon witnesses an abrupt change and presents a configuration that cannot be realized in real folding patterns, which means that now this model experience an unrigid folding. In this paper, we confirm the maximum foldable angle by adjusting the bar in Grasshopper bit by bit and find the critical states in squares, regular hexagons and regular octagons using the folding pattern discussed in this paper.

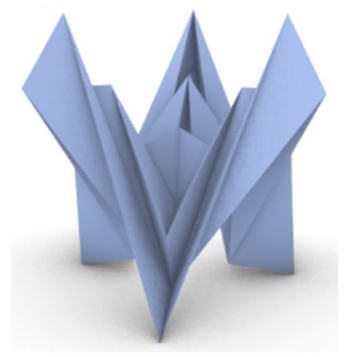

(a)

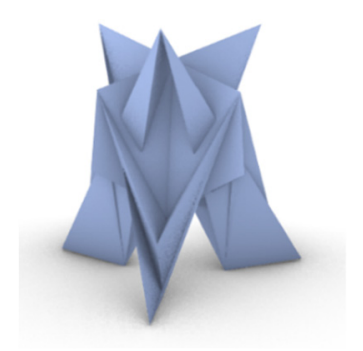

(b)

Figure 19. The perspective observed in Rhinoceros. (a) The folding of a regular hexagon when the folding angle reaches its maximum foldable angle; $(\mathbf{b})$ the perspective of the hexagon when the angle is above the maximum foldable angle.

In this paper, the folding angle is defined as the angle between the sub-plane of each polygon and the $\mathrm{X}-\mathrm{Y}$ plane. According to the authors' observation, in concentric pleating squares which use alternating asymmetric triangulation, there is no abrupt change being observed so that it is concluded that the maximum foldable angle is $90^{\circ}$ for squares. In regular hexagons, the critical state occurs when the angle reaches $79.525^{\circ}$. 


\subsection{The Tessellation of Concentric Pleating Squares}

When there are more than six edges in a regular polygon, it is impossible for this polygon to be tessellated and cover the whole plane. In this case, when discussing the tessellation, this paper just focuses on the square for its simpler structure rather than the hexagon. The tessellation is built through a symmetric copy of one single concentric pleating square, as shown in Figure 20.

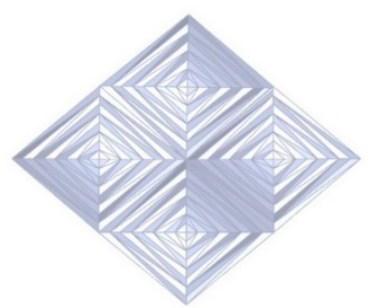

(a)

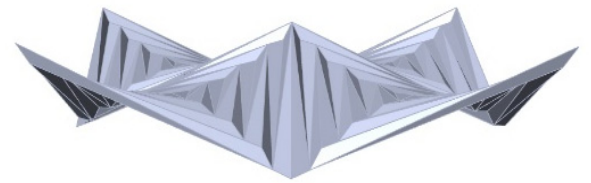

(b)

Figure 20. Tessellation of squares with alternating asymmetric triangulation: (a) Top view; (b) front view.

The tessellation in Figure 20 above has four degrees of freedom and the outlook is unique and artistic. When it is one single square, the fold angle is up to $90^{\circ}$, while here it is around one-third of the former angle, due to the reverse buckling.

As shown in Figure 21, the top vertices in side views are projected inside the projected area. Then, there would be overlaps and collisions among these edges. Consequently, the ultimate state is when the projection of the highest vertex falls on the boundary of the projected area.

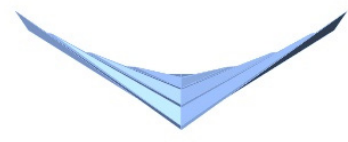

(a)

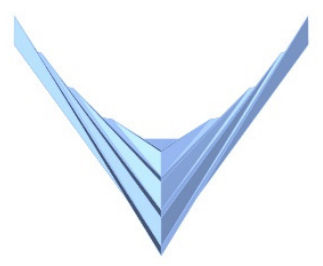

(b)

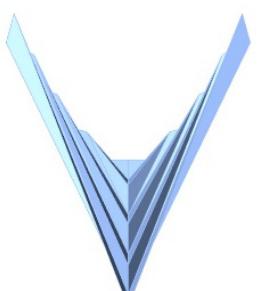

(c)

Figure 21. Reverse buckling of squares with alternating asymmetric triangulation: (a) No buckling; (b) critical state; (c) buckling.

\subsection{Suggestions for Configuration}

Considering the characteristics of this folding pattern, different polygons have various geometric properties that can be applied in different situations. The following are some suggestions for configuration. When a single square is folded based on alternative asymmetric triangulation, the maximum folding angle is $90^{\circ}$ and it has one degree of freedom. Obviously, its tessellation is practical according to the discussion in Section 3.1. For its extremely high folding efficiency, this pattern can be applied when the build asks for high efficiency; meanwhile, the span of this structure is moderate. Compared with squares, triangulated regular hexagons have lower efficiency, especially when there are more than one hexagons tessellated together. However, because of its novel outlook, regular hexagons are helpful if a building demands a unique architectural style. Furthermore, rooves using this pattern will have a larger span. When it comes to a regular octagon, the maximum fold angle of one single octagon is $45^{\circ}$, so the tessellation of octagons must have a comparatively low folding efficiency and this is not feasible in folding. However, its outlook is still inspiring in architecture. It can be concluded that alternating asymmetric triangulation is not suitable for use in polygons with more edges than the polygons given before. 


\section{Conclusions}

The aim of this paper is to illustrate the geometric properties of concentric pleating based on alternating asymmetric triangulation and make use of these characteristics to realize parametric modeling of this folding pattern. During the whole process of kinematic motion, the distances between two adjacent points is immobile, so the authors take advantage of this property to find a feasible and convenient way to generalize the parametric modeling from squares to regular hexagons, using the intersections of some spheres and planes to obtain the coordinates of all the points successively. Though there exists some difference between the motion of squares and that of hexagons, the overall concepts are the same and can be generalized to n-regular polygons in this paper.

These models can be constructed parametrically in the digital environment of Rhinoceros, using Kangaroo in Grasshopper, so that designers can directly observe a model's kinematic deployment and calculate the folding efficiency. The authors make use of the convenience of modeling using Kangaroo in Grasshopper in the environment of Rhinoceros to obtain the maximum foldable angle both for a single polygon and their tessellations. The folding of one single polygon limits the tessellation of several polygons and the available folding angle will be around one-third of that of a single polygon, which diminishes the folding efficiency. Besides, this paper makes conclusions that the tendencies of folding efficiency in different regular polygons are not the same: In squares, the graph is a quadratic line, while in regular hexagons it is a cubic curve. To maintain the rigid folding, each polygon has a limit folding angle, beyond which there will be stretching or teasing within the paper. Meanwhile, the number of creases meeting in each vertex also follows some rules, as shown in Table 1.

Author Contributions: Conceptualization, Q.Z. and D.Y.; Data curation, Q.Z.; Formal analysis, D.Y.; Funding acquisition, F.F.; Investigation, D.Y.; Methodology, D.Y. and X.L.; Project administration, Q.Z.; Resources, F.F.; Software, D.Y. and X.L.; Supervision, F.F.; Validation, Q.Z., X.L. and F.F.; Visualization, D.Y. and X.L.; Writing—original draft, D.Y.; Writing—review \& editing, Q.Z. and D.Y.

Funding: This research was financially supported by the National Natural Science Foundation of China (Grant no. 51978207, 51927813 and 51508132).

Acknowledgments: The authors are grateful to the members of the Space Structures Research Center at the Harbin Institute of Technology for providing invaluable information and advice in this study, and Bajracharya Diwas for editing the manuscript's style and English.

Conflicts of Interest: The authors declare no conflict of interest. The funders had no role in the design of the study; in the collection, analyses, or interpretation of data; in the writing of the manuscript, or in the decision to publish the results.

\section{Appendix A}

The scripts of a regular hexagon using alternating asymmetry triangulation in Grasshopper. See "Origami_hexagon".

\section{References}

1. Gantes, C.J. Deployable Structures: Analysis and Design; National Technical University of Athens: Athens, Greece, 2001.

2. Rogers, J.; Huang, Y.; Schmidt, O.G.; Gracias, D.H. Origami MEMS and NEMS. MRS Bull. 2016, 2, 123-129. [CrossRef]

3. Gracias, D.; Cho, J.-H.; Hu, S. A Three Dimensional Self-folding Package (SFP) for Electronics. MRS Proc. 2010, 1249, F09. [CrossRef]

4. Vergauwen, A.; De Temmerman, N.; Brancart, S. Design and physical modelling of deployable structures based on curved-line folding. In Proceedings of the 4th International Conference on Mobile, Adaptable and Rapidly Assembled Structures, Ostend, Belgium, 11-13 June 2014; WIT Press: Southampton, UK, 2014. [CrossRef]

5. Puig, L.; Barton, A.; Rando, N. A review on large deployable structures for astrophysics missions. Acta Astronaut. 2010, 67, 12-26. [CrossRef] 
6. Naser, M.Z.; Chehab, A.I. Materials and design concepts for space-resilient structures. Prog. Aerosp. Sci. 2018, 98, 74-90. [CrossRef]

7. De Temmerman, N.; Roovers, K.; Alegria Mira, L.; Vergauwen, A.; Koumar, A.; Brancart, S.; De Laet, L.; Mollaert, M. Engineering lightweight transformable structures. In Proceedings of the International Conference on Adaptation and Movement in Architecture, Toronto, ON, Canada, 10-11 October 2013.

8. Gross, D.; Messner, D. The able deployable articulated mast-enabling technology for the shuttle radar topography mission. In Proceedings of the 33rd Aerospace Mechanisms Symposium, Pasadena, CA, USA, 19-21 May 1999.

9. Van Knippenberg, R. Stable Adaptive Structures. Master's Thesis, Eindhoven University of Technology, Eindhoven, The Netherlands, 28 August 2014.

10. Tachi, T. Geometric considerations for the design of rigid origami structures. In Proceedings of the International Association for Shell and Spatial Structures (IASS) Symposium 2010, Shanghai, China, 8-12 November 2010.

11. Tachi, T. Generalization of rigid-foldable quadrilateral-mesh origami. In Proceedings of the International Association for Shell and Spatial Structures (IASS) Symposium 2009, Valencia, Spain, 28 September-2 October 2009.

12. Miura, K. Method of Packaging and Deployment of Large Membranes in Space; Institute of Space and Astronautical Sciences, 1985; Available online: https://repository.exst.jaxa.jp/dspace/handle/a-is/7293 (accessed on 21 September 2019).

13. Sareh, P.; Guest, S.D. Designing Symmetric Derivatives of the Miura-Ori; Advances in Architectural Geometry 2014; Springer: London, UK, 2015; Chapter 15; pp. 233-241. [CrossRef]

14. Sareh, P.; Guest, S.D. Minimal isomorphic symmetric variations on the Miura fold pattern. In Proceedings of the First Conference Transformables 2013, Seville, Spain, 18-20 September 2013.

15. Schenk, M.; Guest, S.D. Geometry of Miura-folded metamaterials. Proc. Natl. Acad. Sci. USA 2013, 110, 3276-3281. [CrossRef] [PubMed]

16. Tachi, T. Designing freeform origami tessellations by generalizing resch's patterns. J. Mech. Des. 2013, 135, 111006. [CrossRef]

17. Resch, R.D. The topological design of sculptural and architectural systems. In Proceedings of the 1973, National Computer Conference and Exposition, New York, NY, USA, 4-8 June 1973; ACM: New York, NY, USA, 1973; pp. 643-650. [CrossRef]

18. Tachi, T. Origamizing polyhedral surfaces. IEEE Trans. Vis. Comput. Graph. 2010, 16, 298-311. [CrossRef] [PubMed]

19. Demaine, E.D.; Demaine, M.L.; Hart, V.; Price, G.N.; Tachi, T. (Non)existence of pleated folds: How paper folds between creases. Gr. Comb. 2011, 27, 377-397. [CrossRef]

20. Tachi, T. Rigid Origami Simulator. Available online: http://www.tsg.ne.jp/TT/software/ (accessed on 28 September 2007).

21. Demaine, E.D.; Tachi, T. Origamizer: A practical algorithm for folding any polyhedron. In Proceedings of the 33rd International Symposium on Computational Geometry, Brisbane, Australia, 4-7 July 2017; pp. 34:1-34:16.

22. Tachi, T. Origamizer. Available online: https://tsg.ne.jp/TT/software/Origamizer047.zip (accessed on 29 August 2008).

23. Tachi, T. Freeform Origami. Available online: https://tsg.ne.jp/TT/software/FreeformOrigami030.zip (accessed on 16 October 2010).

24. Piker, D. Kangaroo. Available online: https://www.grasshopper3d.com/group/kangaroo/ (accessed on 23 May 2015).

25. Grasshopper. Available online: https://www.grasshopper3d.com/ (accessed on 23 May 2008).

26. Robert McNeel \& Associates. Rhinoceros. 2015. Available online: https://www.rhino3d.com/ (accessed on 21 September 2010).

27. Fushimi, K.; Fushimi, M. Origami No Kikagaku (Geometry of Origami); Nihon Hyoronsha: Tokyo, Japan, 1979.

(C) 2019 by the authors. Licensee MDPI, Basel, Switzerland. This article is an open access article distributed under the terms and conditions of the Creative Commons Attribution (CC BY) license (http://creativecommons.org/licenses/by/4.0/). 